\title{
GDF15 tells the brain to lose weight
}

daily
treatment
with murine
and human
recombinant
GDF15 for
4-6 weeks
reduced
food intake,
body weight
and plasma
insulin level,
and improved
metabolic
parameters
in obese mice
and monkeys
$\quad$

Obesity treatments that do not involve invasive surgery are greatly needed. Now, a team of scientists from Amgen provide evidence that harnessing the activity of growth differentiation factor 15 (GDF15; also known as MIC1) can lower the body weight of obese mice and monkeys through effects on the gut-brain axis.

GDF15 had previously been implicated in obesity, insulin resistance and cancer cachexia, and serum levels of GDF15 are increased in obese mice, rats and humans compared with lean controls. However, deletion of $G d f 15$ in mice results in increased body weight, whereas overexpression results in mice with lower body weight and improved metabolic parameters.

Xiong et al. used a microarray assay to identify secreted proteins that were differentially expressed in mouse models of obesity. The authors observed that GDF15 was highly upregulated in the liver and adipose tissue of such mice. Overexpression of GDF15 reduced food intake and body weight and

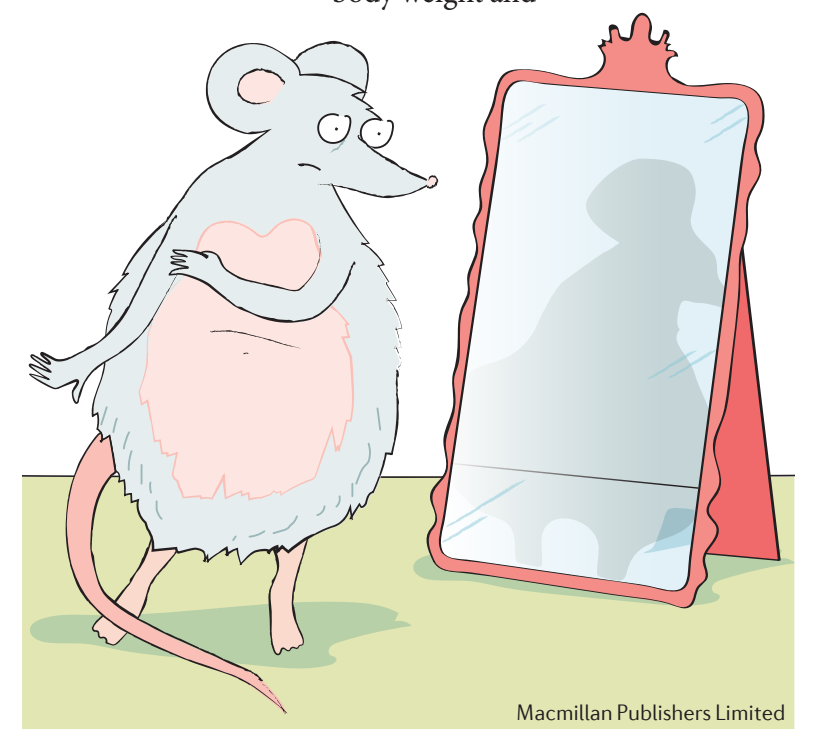

improved metabolic profiles in these mouse models, and mice overexpressing GDF15 were resistant to obesity induced by a high-fat diet. Treatment with an antibody that blocked endogenous GDF15 activity increased body weight and food intake in mice, whereas daily treatment with murine and human recombinant GDF15 for 4-6 weeks reduced food intake, body weight and plasma insulin levels, and improved metabolic parameters in obese mice and monkeys.

However, the use of recombinant GDF15 as a therapeutic is limited by its short serum half-life. To address this, the researchers used an X-ray structure of GDF15 to help design an Fc fusion protein that preserved the biological activity of GDF15 while increasing its yield, half-life and manufacturability. Mice and monkeys treated with the modified form of the protein lost $\sim 10-24 \%$ of their body weight over 5-6 weeks, even while being fed a high-fat diet. Treated animals also showed reductions in key metabolic parameters that are often elevated in obese humans, including lower levels of insulin, glucose and triglycerides.

"Several observations from the study helped to account for the impact on weight loss", says Murielle Veniant, lead author of the study. "Treated mice ate less food and gastric clearance occurred more slowly. They also showed a taste preference for foods that were lower in fat."

The receptor for GDF15 was unknown until just a few months ago, when four groups simultaneously and independently reported that GDF15 binds specifically to GDNF family receptor a-like (GFRAL) and characterized the neuronal circuits through which GDF15 acts (see Further reading). All groups pinpointed the expression of GFRAL in mice to two regions within the hindbrain: the area postrema, which had previously been associated with satiety control and is the only part of the brain outside the blood-brain barrier, and the nucleus of the solitary tract, which receives inputs from the vagus nerve, which mediates gut motility and secretion. Indeed, Xiong et al. found that the delay of gastric emptying induced by GDF15 was not observed in mice after vagotomy but was preserved in sham-operated animals, indicating that GDF15 acts through a vagus-mediated mechanism.

This study provides further evidence of the GDF15-GFRALmediated regulation of food intake by a central mechanism and presents GDF15 Fc fusion proteins as potential therapeutics to target this axis. However, there is still much to learn about this pathway. "For example, why is the protein elevated in animal models of obesity, but injecting animals with this same protein actually leads to weight loss?" says Veriant. "It's also unknown at this point how the protein could influence other findings from the study, such as food preference or changes in triglyceride levels."

\section{Teresa Villanueva}

ORIGINAL ARTICLE Xiong, J. et al. Long-acting MIC-1/GDF15 molecules to treat obesity: evidence from mice to monkeys. Sci. Transl Med. 9 , eaan8732 (2017)

FURTHER READING Yang, L. et al. GFRAL is the receptor for GDF15 and is required for the anti-obesity effects of the ligand. Nat. Med. 23, 1158-1166 (2017) I Mullican, S. E. et al. GFRAL is the receptor for GDF15 and the ligand promotes weight loss in mice and nonhuman primates. Nat. Med. 23,1150-1157 (2017) I Emmerson, P. J. et al. The metabolic effects of GDF15 are mediated by the orphan receptor GFRAL. Nat. Med. 23, 1215-1219 (2017) I Hsu, J.Y. et al. Non-homeostatic body weight regulation through a brainstem-restricted receptor for GDF15. Nature 550, 255-259 (2017) 